\title{
DINÂMICA DE POPULAÇÕES DE ESPÉCIES ARBÓREAS EM FRAGMENTOS DE FLORESTA ALUVIAL NO SUL DE MINAS GERAIS, BRASIL
}

\author{
Matheus Henrique Nunes ${ }^{1}$, Pedro Higuchi ${ }^{2}$, Ana Carolina da Silva ${ }^{2}$, Eduardo van den Berg ${ }^{3}$, \\ Marcela de Castro Nunes Santos ${ }^{4}$ \\ ${ }^{1}$ University of Cambridge, Fitzwilliam College, Cambridge, Inglaterra - matheusfloresta.ptc@hotmail.com \\ ${ }^{2}$ Universidade do Estado de Santa Catarina, Departamento de Engenharia Florestal, Lages, Santa Catarina, Brasil -higuchip@ gmail.com; \\ carol_si14@yahoo.com.br \\ ${ }^{3}$ Universidade Federal de Lavras, Departamento de Biologia, Lavras, Minas Gerais, Brasil - evandenb@dbi.ufla.br \\ ${ }^{4}$ Universidade Federal de Lavras, Programa de Pós-Graduação em Engenharia Florestal, Lavras, Minas Gerais, Brasil - \\ marcelacns@gmail.com
}

Recebido para publicação: 16/12/2013 - Aceito para publicação: 18/12/2015

\begin{abstract}
Resumo
O presente estudo objetivou avaliar a dinâmica de populações arbóreas em fragmentos florestais em São Sebastião da Bela Vista, MG. Para isso, todos os indivíduos arbóreos com diâmetro na altura do peito (DAP) maior do que $5 \mathrm{~cm}$, presentes nas unidades amostrais, foram medidos (DAP e altura) e identificados no ano de 2005 e monitorados nos anos 2007 e 2009 (remedição e verificação de mortalidade), quando também foram medidos os indivíduos que alcançaram o diâmetro mínimo de inclusão por ocasião de um eventual recrutamento. Foram calculadas as taxas de dinâmica anual (mortalidade, recrutamento, rotatividade e mudanças líquidas) para todas as populações. Foi observada instabilidade da dinâmica, indicada pela redução constante da abundância e pelo maior número de espécies com balanço negativo de número de indivíduos (período 2005 a $2007=13$ e 2007 a $2009=17$ ) do que balanço positivo $(2005$ a $2007=9$ e 2007 a $2009=14$ ). As mudanças de riqueza foram insignificantes, com o ganho de duas espécies e o desaparecimento de uma. O estudo demonstrou que, apesar de não apresentar mudanças qualitativas (composição florística), a área demonstrou instabilidade estrutural, com mais populações com maior taxa de mortalidade que de recrutamento.

Palavras-chave: Populações arbóreas; florestas inundáveis; mudanças florístico-estruturais.
\end{abstract}

\begin{abstract}
Tree population dynamics in alluvial forest fragments in south of Minas Gerais, Brazil. The present study aimed to evaluate the tree population dynamics in forest fragments, in the municipality of São Sebastião da Bela Vista, MG. For this sake, all living trees with diameter at breast height (DBH) higher than $5 \mathrm{~cm}$, present in the sampled units, were measured (DBH and height) and identified in 2005 and monitored in 2007 and 2009 (remeasured and mortality evaluation), when all individuals reaching the minimum diameter of inclusion, on the occasion of a possible recruitment, were recruited. The annual dynamics rates (mortality, recruitment, turnover and net change) were calculated for all populations. Dynamics instability was observed, indicated by the steady reduction of abundance and the higher number of species with negative balance in abundance (period 2005 to $2007=13$ and 2007 to $2009=17$ ) than positive balance (2005 to $2007=9$ and 2007 to $2009=14$ ). The richness changes were insignificant, with the gain of two species and loss of just one. The present study demonstrated that, despite the observation of no qualitative (floristic) changes, the evaluated area showed a dynamics with structural instability, with higher mortality than recruitment.

Keywords: Tree population; flooded forests; floristic-structural changes.
\end{abstract}

\section{INTRODUÇÃO}

O monitoramento da dinâmica de comunidades e populações de espécies arbóreas em paisagens frag mentadas é relevante, pois permite um maior entendimento sobre as mudanças florísticas e estruturais que ocorrem ao longo do tempo. Esse conhecimento é fundamental para a avaliação de possíveis efeitos dos impactos causados pela interação sinérgica entre o aquecimento global e o uso da terra pelo homem sobre frag mentos florestais (BRODIE et al., 2012).

Alguns trabalhos têm sido realizados em áreas sob o domínio da Floresta Atlântica com dinâmica florestal, abordando estudos de comunidades e populações arbóreas (OLIVEIRA FILHO et al., 1997; CHA GAS et al., 2001; CORREA; VAN DEN BERG, 2002; OLIVEIRA FILHO et al., 2007; HIGUCHI et al., 2008a;

FLOREST A, Curitiba, PR, v. 46, n. 1, p. 57 - 66, jan. / mar. 2016.

Nunes, M. H. etal.

ISSN eletrônico 1982-4688

DOI: $10.5380 /$ rf.v46il .34782 
HIGUCHI et al., 2008b; MACHADO; OLIVEIRA FILHO, 2010). De forma geral, es ses trabalhos demonstram instabilidade nas taxas de dinâmica, que são influenciadas pelo histórico de perturbação (OLIVEIRA FILHO et al., 1997) e pelo efeito de borda (OLIVEIRA FILHO et al., 2007). Além disso, devido ao processo de silvigênese (MACHADO; OLIVEIRA FILHO, 2010), as taxas de dinâmica não ocorrem de forma constante ao longo do tempo (HIGUCHI et al., 2008a). Porém, poucos trabalhos na região abordam estudos de dinâmica em ambientes aluviais (GUILHERME et al., 2004; APPOLINÁ RIO et al., 2005; GUIMA RÃ ES et al., 2008; SILVA et al., 2011a; SILVA et al., 2011b), o que justifica estudos nesses ambientes, por permitirem gerar conhecimentos que podem subsidiar estratégias de manejo e restauração florestal nesses locais.

No ano de 2005, Silva et al. (2009) inventariaram um fragmento de floresta ciliar e cinco frag mentos localizados na planície aluvial em São Sebastião da Bela Vista, MG. A primeira dinâmica das comunidades nesses fragmentos foi estudada por Silva et al. (2011a), compreendendo o período de 2005-2007, que coincidiu com uma enchente de grandes proporções ocorrida em janeiro de 2007 na região. Também foi estudada a segunda dinâmica da comunidade arbórea, abrangendo o período de 2007-2009 (SILVA et al., 2011b). Verificou-se a necessidade de avaliação da variação das taxas de dinâmica das populações arbóreas em ambos os períodos, possibilitando avaliar as estratégias dessas espécies frente às mudanças ambientais às quais estão sujeitas. Dessa forma, este trabalho objetivou avaliar a dinâmica das populações arbóreas presentes em frag mentos de Floresta Aluvial no sul de Minas Gerais.

\section{MATERIAL E MÉTODOS}

\section{Descrição da área de estudo}

O estudo foi realizado em frag mentos florestais localizados no município de São Sebastião da Bela Vista, Minas Gerais. Foram estudados cinco fragmentos florestais da planície aluvial adjacente ao rio Sapucaí e um fragmento de floresta ciliar sobre o dique do mesmo rio (Figura 1). A região estudada está localizada na Bacia Hidrográfica do Rio Sapucaí, que integra a Bacia do Rio Grande, nas coordenadas geográficas de $22^{\circ} 05^{\prime} 57^{\prime \prime} \mathrm{S}$ a $22^{\circ} 07^{\prime} 22,5^{\prime \prime} \mathrm{S}$ e $45^{\circ} 48^{\prime} 05^{\prime \prime} \mathrm{W}$ a $45^{\circ} 48^{\prime} 53,5^{\prime \prime} \mathrm{W}$, sendo a altitude média dos fragmentos avaliados de $809 \mathrm{~m}$.

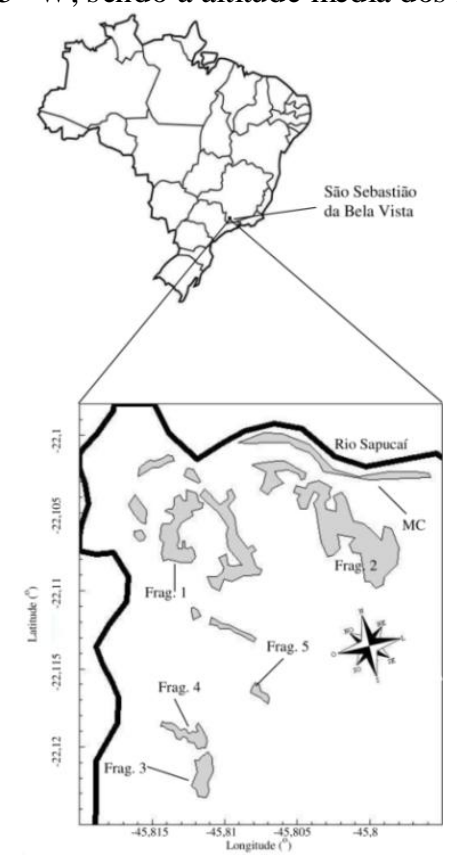

Figura 1. Floresta ciliar (MC) e fragmentos florestais estudados em uma floresta aluvial no município de São Sebastião da Bela Vista, MG. A representação do rio Sapucaí aparece em preto, os fragmentos florestais presentes na área estão em cinza e, em branco, está representada a matriz não florestal. Fonte: Silva et al., 2009.

Figure 1. Riparian forest (MC) and forest fragments studied in an alluvial forest in São Sebastião da Bela Vista, MG. Sapucaí river is represented in black, forest fragments are in gray and white indicates the nonforest matrix. Source: Silva et al., 2009.

O relevo predominante varia entre o plano e o suave ondulado, sendo que as formas planas estão sujeitas a inundações periódicas, com enchentes de diferentes intensidades. Os solos da região são, predominantemente, Neossolos na floresta ciliar, Gleissolos nas áreas de maior encharcamento e Cambissolos 
nas áreas menos saturadas dos fragmentos (SILVA et al., 2009). Segundo a classificação de Köppen, o clima predominante na região é mesotérmico úmido, com dois a três meses secos ao ano, enquadrado no tipo Cwb (temperatura média do mês mais frio inferior a $18{ }^{\circ} \mathrm{C}$ e do mês mais quente não ultrapassando $22^{\circ} \mathrm{C}$ ) (BRASIL, 1992). O índice pluviométrico anual está entre 1.300 e $1.700 \mathrm{~mm}$, com o período seco nos meses de junho a agosto, e o mais chuvoso de dezembro a fevereiro, concentrando $50 \%$ da pluviosidade anual. A umidade relativa do ar varia entre 70 e $82 \%$ durante o ano, sendo os valores mais baixos observados nos meses de junho a agosto. A região sul mineira enquadra-se em zona de influência dos domínios vegetacionais do cerrado e da Floresta Atlântica (RIZZINI, 1997) e as florestas da área de estudos são classificadas como Floresta Estacional Semidecidual Aluvial (INSTITUTO BRASILEIRO DE GEOGRAFIA E ESTATÍSTICA, 2012).

A floresta aluvial estudada foi inventariada por Silva et al. (2009) em julho de 2005 e teve a dinâmica de sua comunidade avaliada em dois períodos: 2005-2007 (SILVA et al., 2011a) e 2007-2009 (SILVA et al., 2011 b). O primeiro período de dinâmica, avaliado em julho de 2007, teve seu estudo realizado seis meses após uma enchente de grandes proporções, ocorrida no mês de janeiro, durante uma época de grande pluviosidade na região, quando as árvores da floresta ciliar e de alguns fragmentos ficaram parcialmente submersas, o que não impediu a realização das avaliações, visto que no mês de julho o nível da água retornou ao seu nível mais baixo, como padrão esperado da época mais seca.

\section{Amostragem da vegetação}

Os estudos de dinâmica foram conduzidos nas 54 parcelas de $200 \mathrm{~m}^{2}(10$ x $20 \mathrm{~m}$ ) alocadas em 2005 no estudo fitossossiológico de Silva et al. (2009), sendo 24 parcelas alocadas na floresta ciliar e seis em cada um dos cinco fragmentos avaliados, distribuídas de forma sistemática nos locais. No primeiro inventário em 2005 , todos os indivíduos arbóreos vivos dentro das parcelas que apresentaram diâmetro medido a 1,30 m de altura (DAP) igual ou superior a $5 \mathrm{~cm}$ foram identificados e mensurados (DAP e altura). As árvores com caules mú ltip los foram medidas quando a soma das áreas basais das secções dos caules correspondeu a uma área basal igual ou maior que a de um caule único com $5 \mathrm{~cm}$ de DAP. As identificações foram realizadas por meio de consultas a especialista (Sobral, M. E. G.) e todo o material testemunha está incorporado no herbário da Universidade Federal de Lavras (ESAL), conforme consta no trabalho de Silva et al. (2009). Os inventários posteriores, no ano de 2007 e de 2009, seguiram a mesma metodologia, incorporando-se a identificação e mensuração dos novos indivíduos que atingiram o DAP $\geq 5 \mathrm{~cm}$. Os indivíduos mortos, em pé ou caídos, foram registrados. Os sobreviventes observados durante os anos de avaliação foram mensurados novamente.

\section{Análise dos dados}

Foram calculadas, nos períodos 2005-2007 e 2007-2009, as taxas anuais de mortalidade (M) e recrutamento $(\mathrm{R})$, com base no número de indivíduos, por meio dos modelos algébricos propostos por Lieberman et al. (1985), Primack et al. (1985) e Sheil e May (1996):

$$
\begin{aligned}
& M=\left[1-\left(\frac{N o-N m}{N o}\right)^{\frac{1}{t}}\right] \times 100 ; \\
& R=\left\{1-\left[1-\left(\frac{N r}{N f}\right)^{\frac{1}{t}}\right]\right\} \times 100 ;
\end{aligned}
$$

em que: $M=$ taxa de mortalidade $\left(\% \cdot\right.$ ano $\left.^{-1}\right) ; N o=$ número de indivíduos no primeiro levantamento; $N m=$ nú mero de indivíduos mortos; $t=$ intervalo em anos entre levantamentos; $R=$ taxa de recrutamento $\left(\%\right.$. ano $\left.^{-1}\right)$; $N r=$ nú mero de indivíduos recrutados; e $N f=$ número de indivíduos no último levantamento.

A taxa média anual de rotatividade (turnover) em nú mero de árvores (Tn), que expressa a dinâmica global das populações (COSTA et al., 2010), foi calculada pela média das taxas de mortalidade e de recrutamento (KORNING; BALSLEV, 1994; OLIVEIRA FILHO et al., 1997):

$$
\operatorname{Tn}=\frac{R+M}{2}
$$

Conforme Korning e Balslev (1994), foram obtidas as taxas anuais de mudança líquida para o nú mero de árvores (Chn), sendo utilizada a equação:

FLOREST A, Curitiba, PR, v. 46, n. 1, p. 57 - 66, jan. / mar. 2016.

Nunes, M. H. et al.

ISSN eletrônico 1982-4688

DOI: $10.5380 /$ rf.v46il .34782 


$$
\operatorname{Chn}=\left[\left(\frac{N f}{N o}\right)^{\frac{1}{t}}-1\right] \times 100 .
$$

As taxas de mudanças líquidas sintetizam a relação entre a mortalidade e o recrutamento de uma população, de forma que valores positivos indicam recrutamento superior à mortalidade e valores negativos indicam mortalidade superior ao recrutamento. Ainda, de acordo Korning e Balslev (1994), a taxa anual de mudança líquida é calculada as sumindo-se que mortalidade e recruta mento de árvores constituem u ma proporção constante da população inicial por intervalo de tempo.

Os dados foram processados no programa R, (R DEVELOPMENT CORETEAM, 2013).

\section{RES ULTADOS}

As sete espécies que se mantiveram com maior abundância nos três períodos avaliados foram (Tabela 1): Sebastiania commersoniana, Guarea macrophylla, Inga vera, Casearia sylvestris, Nectandra nitidula, Alchornea triplinervia e Eugenia florida. Por outro lado, sete espécies ocorreram de forma rara (um indivíduo) no primeiro inventário e permaneceram assim durante todo o tempo monitorado, sem recrutamento e sem mort alidade: Andira fraxinifolia, Campomanesia guaviroba, Cryptocarya saligna, Erythrina falcata, Ficus luschnathiana, Lacistema hasslerianum e Miconia paulensis. Duas novas espécies foram recrutadas: Nectandra lanceolata, com dois indivíduos, e Myrsine coriacea, com um indivíduo. Uma espécie desapareceu (Sorocea bonplandii), com a mortalidade do único indivíduo existente. Ess as últimas mudanças ocorreram no terceiro inventário.

Tabela 1. Taxas de dinâmica das populações arbóreas, ordenadas de forma decrescente pelo número de indivíduos em 2009, nos períodos de 2005-2007 e 2007-2009, em u ma floresta aluvial no mun icípio de São Sebastião da Bela Vista, MG. N representa o número de indivíduos para os anos. M (taxa de mortalidade), $\mathrm{R}$ (taxa de recrutamento), Tn (taxa de rotatividade) e Chn (taxa de mudança líquida em densidade) representam as taxas para cada espécie, express as em $\%$.ano- ${ }^{-1}$.

Table 1. Dynamics rates of tree populations, sorted in decreasing order of number of individuals in 2009, for the periods of 2005-2007 and 2007-2009, in an alluvial forest in São Sebastião da Bela Vista, MG. N represents the number of individuals for the years. $\mathrm{M}$ (mortality rate), $\mathrm{R}$ (recruitment rate), $\mathrm{Tn}$ (turnover rate) and Chn (net change rate) represent the dynamics rates, expressed as \%.year ${ }^{-1}$.

\begin{tabular}{|c|c|c|c|c|c|c|c|c|c|c|c|}
\hline \multirow{2}{*}{ Espécies } & \multirow{2}{*}{$\underset{2005}{N}$} & \multirow{2}{*}{$\underset{2007}{N}$} & \multirow{2}{*}{$\underset{2009}{N}$} & \multicolumn{4}{|c|}{$2005-2007$} & \multicolumn{4}{|c|}{ 2007-2009 } \\
\hline & & & & $\mathbf{M}$ & $\mathbf{R}$ & Tn & Chn & $\mathbf{M}$ & $\mathbf{R}$ & Tn & Chn \\
\hline $\begin{array}{l}\text { Sebastiania commersoniana } \\
\text { (Baill.) L.B.Sm. \& Downs }\end{array}$ & 851 & 825 & 787 & 1,9 & 0,4 & 1,1 & $-1,5$ & 2,5 & 0,2 & 1,4 & $-2,3$ \\
\hline Guareamacrophylla Vahl & 285 & 296 & 305 & 0,7 & 2,6 & 1,6 & 1,9 & 0,8 & 2,3 & 1,6 & 1,5 \\
\hline Inga vera Willd. & 220 & 203 & 173 & 3,9 & 0,0 & 2,0 & $-3,9$ & 8,0 & 0,3 & 4,1 & $-7,7$ \\
\hline Casearia sylvestris Sw. & 93 & 95 & 92 & 0,5 & 1,6 & 1,1 & 1,1 & 3,2 & 1,6 & 2,4 & $-1,6$ \\
\hline Nectandra nitidula Nees & 73 & 72 & 65 & 2,1 & 1,4 & 1,7 & $-0,7$ & 5,7 & 0,8 & 3,2 & $-5,0$ \\
\hline $\begin{array}{l}\text { Alchomea triplinervia } \\
\text { (Spreng.) Müll.Arg. }\end{array}$ & 63 & 64 & 63 & 0,0 & 0,8 & 0,4 & 0,8 & 2,4 & 1,6 & 2,0 & $-0,8$ \\
\hline Eugenia florida DC. & 33 & 35 & 40 & 0,0 & 2,9 & 1,4 & 3,0 & 0,0 & 6,5 & 3,2 & 6,9 \\
\hline $\begin{array}{l}\text { Nectandra megapotamica } \\
\text { (Spreng.) Mez }\end{array}$ & 30 & 30 & 30 & 1,7 & 1,7 & 1,7 & 0,0 & 1,7 & 1,7 & 1,7 & 0,0 \\
\hline Picramnia sellowii Planch. & 30 & 30 & 30 & 0,0 & 0,0 & 0,0 & 0,0 & 1,7 & 1,7 & 1,7 & 0,0 \\
\hline $\begin{array}{l}\text { Annona emarginata } \\
\text { (Schltdl.) H.Rainer }\end{array}$ & 31 & 28 & 27 & 5,0 & 0,0 & 2,5 & $-5,0$ & 3,6 & 1,9 & 2,8 & $-1,8$ \\
\hline Copaifera lang sdorffii Desf. & 24 & 26 & 27 & 0,0 & 3,9 & 2,0 & 4,1 & 0,0 & 1,9 & 0,9 & 1,9 \\
\hline $\begin{array}{l}\text { Pouteria gardneriana } \\
\text { (A.DC.) Radlk. }\end{array}$ & 21 & 21 & 21 & 0,0 & 0,0 & 0,0 & 0,0 & 0,0 & 0,0 & 0,0 & 0,0 \\
\hline $\begin{array}{l}\text { Vitex megapotamica } \\
\text { (Spreng.) Moldenke }\end{array}$ & 25 & 24 & 21 & 4,1 & 2,1 & 3,1 & $-2,0$ & 6,5 & 0,0 & 3,2 & $-6,5$ \\
\hline $\begin{array}{l}\text { Eugenia dodonaeifolia } \\
\text { Cambess. }\end{array}$ & 17 & 18 & 19 & 0,0 & 2,8 & 1,4 & 2,9 & 2,8 & 5,4 & 4,1 & 2,7 \\
\hline Eugenia sp. & 16 & 16 & 18 & 0,0 & 0,0 & 0,0 & 0,0 & 0,0 & 5,7 & 2,9 & 6,1 \\
\hline $\begin{array}{l}\text { Symplocos uniflora (Pohl) } \\
\text { Benth. }\end{array}$ & 22 & 19 & 17 & 7,1 & 0,0 & 3,5 & $-7,1$ & 5,4 & 0,0 & 2,7 & $-5,4$ \\
\hline $\begin{array}{l}\text { Calyptranthes widgreniana } \\
\text { O.Berg }\end{array}$ & 18 & 18 & 16 & 0,0 & 0,0 & 0,0 & 0,0 & 5,7 & 0,0 & 2,9 & $-5,7$ \\
\hline $\begin{array}{l}\text { Symplocos celastrinea } \\
\text { Mart. ex Miq. }\end{array}$ & 26 & 25 & 16 & 3,9 & 2,0 & 3,0 & $-1,9$ & 20,0 & 0,0 & 10,0 & $-20,0$ \\
\hline
\end{tabular}




\begin{tabular}{|c|c|c|c|c|c|c|c|c|c|c|c|}
\hline Tapirira guianensis Aubl. & 16 & 15 & 16 & 3,2 & 0,0 & 1,6 & $-3,2$ & 0,0 & 3,2 & 1,6 & 3,3 \\
\hline $\begin{array}{l}\text { Myrcia pulchra (O.Berg) } \\
\text { Kiaersk. }\end{array}$ & 12 & 12 & 15 & 0,0 & 0,0 & 0,0 & 0,0 & 0,0 & 10,6 & 5,3 & 11,8 \\
\hline $\begin{array}{l}\text { Maytenus salicifolia } \\
\text { Reissek }\end{array}$ & 10 & 12 & 12 & 0,0 & 8,7 & 4,4 & 9,5 & 0,0 & 0,0 & 0,0 & 0,0 \\
\hline Myrcia oblongata DC. & 12 & 12 & 12 & 0,0 & 0,0 & 0,0 & 0,0 & 0,0 & 0,0 & 0,0 & 0,0 \\
\hline Myrcia undulata O.Berg & 11 & 10 & 10 & 4,7 & 0,0 & 2,3 & $-4,7$ & 5,1 & 5,1 & 5,1 & 0,0 \\
\hline Myrsine gardneriana A.DC. & 11 & 11 & 9 & 0,0 & 0,0 & 0,0 & 0,0 & 20,2 & 11,8 & 16,0 & $-9,5$ \\
\hline Croton urucurana Baill. & 29 & 21 & 9 & 14,9 & 0,0 & 7,5 & $-14,9$ & 34,5 & 0,0 & 17,3 & $-34,5$ \\
\hline $\begin{array}{l}\text { Duguetia lanceolata A.St.- } \\
\text { Hil. }\end{array}$ & 7 & 7 & 8 & 0,0 & 0,0 & 0,0 & 0,0 & 0,0 & 6,5 & 3,2 & 6,9 \\
\hline $\begin{array}{l}\text { Myrciaria tenella (DC.) } \\
\text { O.Berg }\end{array}$ & 6 & 6 & 7 & 0,0 & 0,0 & 0,0 & 0,0 & 0,0 & 7,4 & 3,7 & 8,0 \\
\hline $\begin{array}{l}\text { Syag ris romanzoffiana } \\
\text { (Cham.) Glassman }\end{array}$ & 7 & 7 & 7 & 0,0 & 0,0 & 0,0 & 0,0 & 0,0 & 0,0 & 0,0 & 0,0 \\
\hline $\begin{array}{l}\text { Cupania ludowigii Somner } \\
\text { \& Ferruci }\end{array}$ & 6 & 6 & 6 & 0,0 & 0,0 & 0,0 & 0,0 & 0,0 & 0,0 & 0,0 & 0,0 \\
\hline $\begin{array}{l}\text { Andira vermifuga (Mart.) } \\
\text { Benth. }\end{array}$ & 5 & 4 & 5 & 10,6 & 0,0 & 5,3 & $-10,6$ & 0,0 & 10,6 & 5,3 & 11,8 \\
\hline $\begin{array}{l}\text { Cecropia pachystachya } \\
\text { Trécul }\end{array}$ & 6 & 6 & 5 & 8,7 & 8,7 & 8,7 & 0,0 & 8,7 & 0,0 & 4,4 & $-8,7$ \\
\hline $\begin{array}{l}\text { Eugenia blastantha } \\
\text { (O.Berg) D.Legrand }\end{array}$ & 4 & 5 & 5 & 0,0 & 10,6 & 5,3 & 11,8 & 0,0 & 0,0 & 0,0 & 0,0 \\
\hline $\begin{array}{l}\text { Eugenia cf. myrciariifolia } \\
\text { Soares-Silva \& Sobral }\end{array}$ & 5 & 5 & 5 & 0,0 & 0,0 & 0,0 & 0,0 & 0,0 & 0,0 & 0,0 & 0,0 \\
\hline $\begin{array}{l}\text { Calycorectes psidiiflorus } \\
\text { (O.Berg) Sobral }\end{array}$ & 5 & 5 & 4 & 0,0 & 0,0 & 0,0 & 0,0 & 10,6 & 0,0 & 5,3 & $-10,6$ \\
\hline Trichilia pallida Sw. & 3 & 3 & 4 & 0,0 & 0,0 & 0,0 & 0,0 & 0,0 & 13,4 & 6,7 & 15,5 \\
\hline $\begin{array}{l}\text { Daphnopsis fasciculata } \\
\text { (Meisn.) Nevling }\end{array}$ & 3 & 3 & 3 & 0,0 & 0,0 & 0,0 & 0,0 & 0,0 & 0,0 & 0,0 & 0,0 \\
\hline $\begin{array}{l}\text { Guettarda vibumoides } \\
\text { Cham. \& Schltdl. }\end{array}$ & 4 & 3 & 3 & 13,4 & 0,0 & 6,7 & $-13,4$ & 0,0 & 0,0 & 0,0 & 0,0 \\
\hline $\begin{array}{l}\text { Myrcia lamotteana } \\
\text { Cambess. }\end{array}$ & 2 & 2 & 3 & 0,0 & 0,0 & 0,0 & 0,0 & 0,0 & 18,4 & 9,2 & 22,5 \\
\hline $\begin{array}{l}\text { Myrcia multiflora (Lam.) } \\
\text { DC. }\end{array}$ & 3 & 3 & 3 & 0,0 & 0,0 & 0,0 & 0,0 & 0,0 & 0,0 & 0,0 & 0,0 \\
\hline $\begin{array}{l}\text { Ocotea indecora (Schott) } \\
\text { Mez }\end{array}$ & 2 & 3 & 3 & 0,0 & 18,4 & 9,2 & 22,5 & 0,0 & 0,0 & 0,0 & 0,0 \\
\hline Xylosma venosa N.E.Brown & 6 & 5 & 3 & 8,7 & 0,0 & 4,4 & $-8,7$ & 36,8 & 18,4 & 27,6 & $-22,5$ \\
\hline $\begin{array}{l}\text { Pera glabrata (Schott) } \\
\text { Poepp. ex Baill. }\end{array}$ & 2 & 2 & 2 & 0,0 & 0,0 & 0,0 & 0,0 & 0,0 & 0,0 & 0,0 & 0,0 \\
\hline Nectandra lanceolata Nees & 0 & 0 & 2 & - & - & - & - & - & 100,0 & - & - \\
\hline $\begin{array}{l}\text { Plinia cauliflora (Mart.) } \\
\text { Kausel }\end{array}$ & 2 & 2 & 2 & 0,0 & 0,0 & 0,0 & 0,0 & 0,0 & 0,0 & 0,0 & 0,0 \\
\hline Andira fraxinifolia Benth. & 1 & 1 & 1 & 0,0 & 0,0 & 0,0 & 0,0 & 0,0 & 0,0 & 0,0 & 0,0 \\
\hline $\begin{array}{l}\text { Campomanesia guaviroba } \\
\text { (DC.) Kiaersk. }\end{array}$ & 1 & 1 & 1 & 0,0 & 0,0 & 0,0 & 0,0 & 0,0 & 0,0 & 0,0 & 0,0 \\
\hline Cryptocarya saligna $\mathrm{Mez}$ & 1 & 1 & 1 & 0,0 & 0,0 & 0,0 & 0,0 & 0,0 & 0,0 & 0,0 & 0,0 \\
\hline Erythrina falcata Benth. & 1 & 1 & 1 & 0,0 & 0,0 & 0,0 & 0,0 & 0,0 & 0,0 & 0,0 & 0,0 \\
\hline $\begin{array}{l}\text { Ficus luschnathiana (Miq.) } \\
\text { Miq. }\end{array}$ & 1 & 1 & 1 & 0,0 & 0,0 & 0,0 & 0,0 & 0,0 & 0,0 & 0,0 & 0,0 \\
\hline $\begin{array}{l}\text { Lacistema hasslerianum } \\
\text { Chodat }\end{array}$ & 1 & 1 & 1 & 0,0 & 0,0 & 0,0 & 0,0 & 0,0 & 0,0 & 0,0 & 0,0 \\
\hline Miconia paulensis Naudin & 1 & 1 & 1 & 0,0 & 0,0 & 0,0 & 0,0 & 0,0 & 0,0 & 0,0 & 0,0 \\
\hline $\begin{array}{l}\text { Myrsine coriacea (Sw.) } \\
\text { Roem. \& Schult. }\end{array}$ & 0 & 0 & 1 & - & - & - & - & - & 100,0 & - & - \\
\hline $\begin{array}{l}\text { Xylosma prockia (T urcz.) } \\
\text { Turcz. }\end{array}$ & 2 & 2 & 1 & 0,0 & 0,0 & 0,0 & 0,0 & 29,3 & 0,0 & 14,6 & $-29,3$ \\
\hline $\begin{array}{l}\text { Sorocea bonplandii (Baill.) } \\
\text { W.C.Burger et al. }\end{array}$ & 1 & 1 & 0 & 0,0 & 0,0 & 0,0 & 0,0 & 100,0 & 0,0 & - & $-100,0$ \\
\hline Total de indivíduos & 2067 & 2025 & 1939 & & & & & & & & \\
\hline Total de espécies & 52 & 52 & 53 & & & & & & & & \\
\hline
\end{tabular}

Em termos absolutos, foi observada a redução do número de indivíduos na área $(2067,2025,1939)$ e estabilidade da riqueza $(52,52,53)$. Além disso, para ambos os períodos, o número de espécies que apresentou balanço negativo de densidade, ou seja, mortalidade superior ao recrutamento (2005 a $2007=13$ e 2007 a 2009 = 17), foi maior do que o número de espécies combalanço positivo (2005 a 2007 = 9 e 2007 a $2009=14$ ).

FLOREST A, Curitiba, PR, v. 46, n. 1, p. 57 - 66, jan. / mar. 2016 

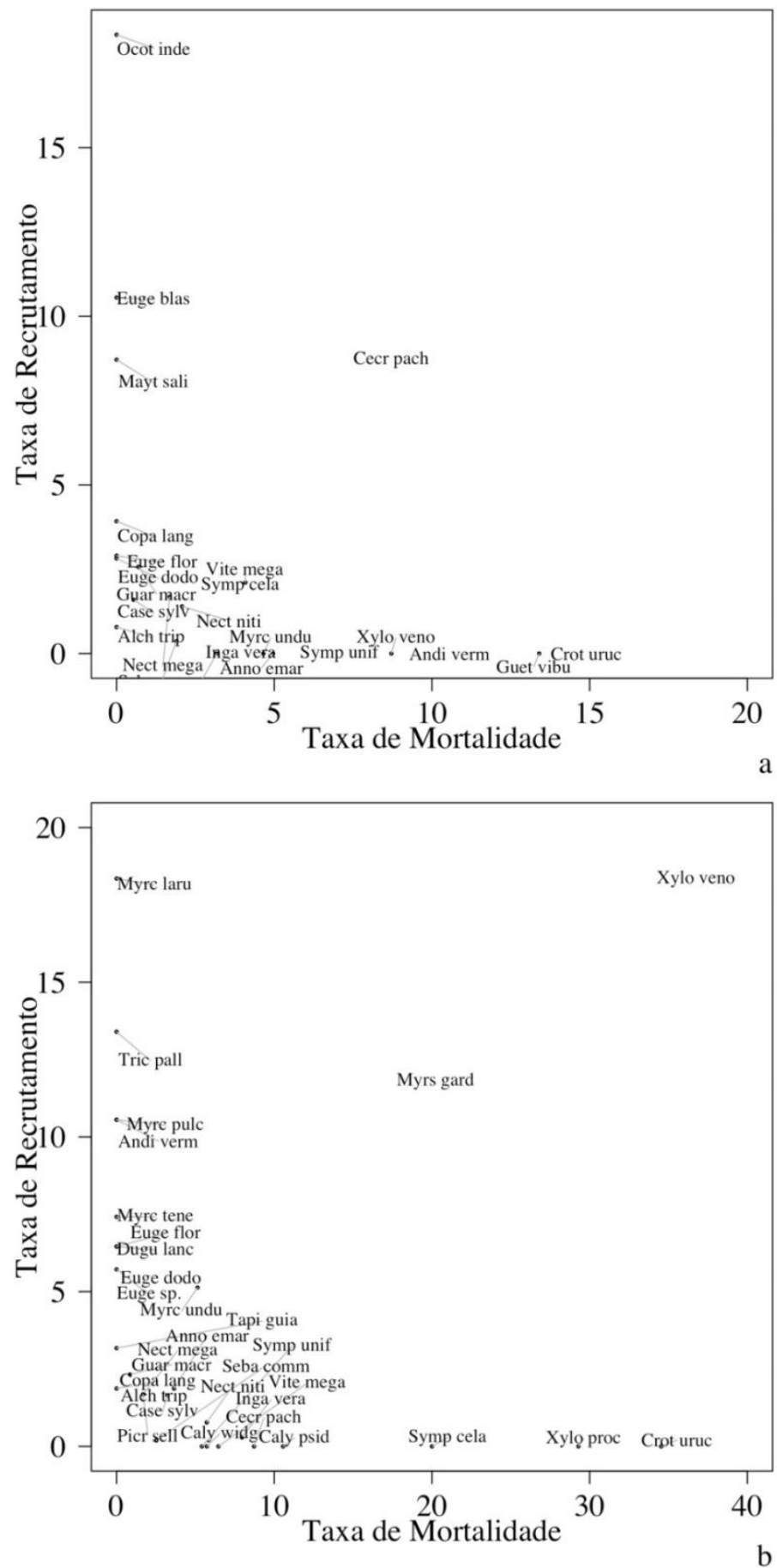

Figura 2. Relação entre as taxas de mortalidade (eixo X) e as taxas de recrutamento (eixo Y), expressas em

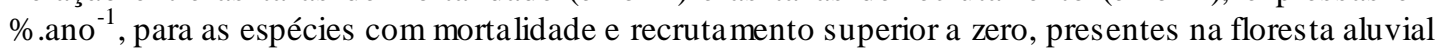
no município de São Sebastião da Bela Vista, MG, no período de 2005-2007 (a) e 2007-2009 (b).

Figure 2. Mortality (x-axis) and recruitment (y-axis) rate relationship of species with mortality and recruitment greater than zero, expressed in \%.year ${ }^{-1}$, in an alluvial forest in the municipality of São Sebastião da Bela Vista, MG, during 2005-2007 (a) and 2007-2009 (b ) period. 
Entre o primeiro e o último levantamento, os maiores valores de indivíduos mortos foram observados para Sebastiania commersoniana $(\mathrm{N}(2005)-\mathrm{N}(2009)=851-787=64)$ e Inga vera $(47)$. No entanto, a espécie com as maiores taxas de mortalidade, considerando-se os dois intervalos de monitoramento, foi Croton urucurana $\left(14,9\right.$ e $34,5 \%$. ano $^{-1}$, respectivamente). Além disso, essa espécie não apresentou recrutamento, o que acabou resultando nas maiores mudanças líquidas observadas nos dois períodos, entre as populações analisadas: $-14,9 \%$.ano ${ }^{-1}$ no primeiro período e $-34,5 \%$.ano ${ }^{-1}$ no segundo período, de forma que a população passou de 29 indivíduos, em 2005, para apenas nove, em 2009.

Além de $C$. urucurana, destacaram-se pela elevada taxa de mortalidade e ausência de recrutamento, em pelo menos um dos períodos avaliados, espécies como Andira vermifuga, Guettarda viburnoides e Xylosma venosa, no prime iro período (Figura 2a), e Xylosma prockia, Symplocos celastrinea e Cecropia pachystachya no segundo período (Figura 2b), resultando em elevada mudança líquida negativa para essas espécies nos respectivos períodos (Tabela 1 ).

Em relação às taxas de recrutamento, Ocotea indecora, Eugenia blastantha (ambas no primeiro período de dinâmica, Figura 2a), Myrcia laruotteana e Trichilia pallida (no segundo período, Figura 2b) apresentaram elevada taxa de recrutamento e nenhuma mortalidade, resultando em taxas de mudança líquida positiva $\left(22,5 \%\right.$.ano ${ }^{-1}$ no primeiro período para O. indecora, $11,8 \%$.ano ${ }^{-1}$ no primeiro período para E. blastantha, $22,5 \%$.ano $^{-1}$ no segundo período para $M$. laruotteana e $15,5 \%$.ano ${ }^{-1}$ no segundo período para $T$. pallida) (Tabela 1). Vale ressaltar que essas espécies apresentaram o incremento de somente um indivíduo durante o estudo, sendo que as elevadas taxas de recrutamento são explicadas pela baixa densidade populacional de las na área. Destaca-se, porém, que, mes mo com baixas taxas demográficas, algumas populações, como, por exe mplo, Sebastiania commersoniana e Guarea macrophylla, são relevantes para a estrutura da floresta, uma vez que apresentam e levado número de indivíduos.

\section{DISCUSSÃO}

Considerando a comunidade como um todo, no que se refere à florística e riqueza, as mudanças foram insignificantes, com ganho apenas de uma espécie durante quatro anos. O mesmo resultado tem sido observado em outros estudos em diferentes fitofisionomias e condições ambientais, que avaliaram a dinâmica florestal em um período curto de tempo (< 15 anos) (PINTO; HAY, 2005; GUIMARÃES et al., 2008; SILVA; ARAÚJO, 2009; MACHADO; OLIVEIRA FILHO, 2010; CARVALHO; FELFILI, 2011; SAITER et al., 2011), o que sugere que esse é um padrão recorrente em áreas tanto inundáveis quanto não inundáveis. Maior estabilidade florística, ou seja, pequena mudança no número de espécies, seria esperada em áreas em avançado estado de sucessão, com baixa intensidade de distúrbios, o que não é o caso da floresta estudada, sujeita a enchentes periódicas e à antropização (corte seletivo ilegal e presença de gado). A partir de estudos sobre trajetórias sucessionais em florestas tropicais (e.g. MASSOCA et al., 2012), infere-se que a velocidade das mudanças florísticas e na riqueza de uma floresta seja maior durante as fases iniciais e intermediárias do processo de sucessão, uma vez que é nes se período que geralmente ocorre a substituição das guildas de espécies mais in iciais por aquelas mais tardias. No caso de ausência de distúrbios de grandes proporções, a velocidade das mudanças diminui, tendendo a uma maior estabilidade relativa em florestas em avançado estágio de sucessão. Dessa forma, a baixa taxa de mudança observada no presente estudo pode ser explicada pelo curto espaço de tempo avaliado e pela interação de dois fatores: i) a homogeneização biológica que ocorre em frag mentos florestais sujeitos a um histórico processo de perturbação antrópica (LÔBO et al., 2011) e ii) a elevada seletividade ambiental de áreas com influência aluvial (SILVA et al., 2010). A homogeneização biológica é representada pela redução do pool de espécies com o potencial de colonizar a floresta, e a elevada seletividade ambiental é representada pela limitação do número de espécies com a capacidade de se estabelecer na área. Assim, a interação entre esses dois fatores poderia resultar na baixa mudança florística ao longo do tempo.

No que refere ao número de indivíduos, a dinâmica revelou um quadro de instabilidade, tan to para a comunidade como um todo quanto para as populações avaliadas, ilustrada pela redução do número de indivíduos e grande parte das espécies com balanço negativo de densidade. A redução na densidade de árvores tem sido observada em vários estudos de dinâmica em florestas na região do Alto Rio Grande (APPOLINÁRIO et al., 2005; HIGUCHI et al., 2008a; MIGUEL et al., 2011). Essa instabilidade pode ser interpretada como sendo o resultado de uma resposta pós-distúrbio, em decorrência do regime de impactos causados pelas cheias de curso d'água (GUILHERME et al., 2004) e pela pressão de origem antrópica (OLIVEIRA FILHO et al., 1997), ambos presentes na área de estudo. A redução de indivíduos também pode ser interpretada como sendo parte de um processo cíclico, representado por diferentes fases de silvegênese (MACHADO; OLIVEIRA FILHO, 2010), sendo que, a partir desse conceito, cada mancha dentro da floresta em diferente estádio sucessional é definida como constituindo uma eco-unidade com diferente tamanho e composição de espécies (BOTREL et al., 2013).

FLOREST A, Curitiba, PR, v. 46, n. 1, p. 57 - 66, jan. / mar. 2016.

Nunes, M. H. etal.

ISSN eletrônico 1982-4688

DOI: $10.5380 / \mathrm{rf} . v 46 \mathrm{il} .34782$ 
Dessa forma, a instabilidade encontrada está associada à substituição de espécies pioneiras, representadas principalmente por $C$. urucurana, por espécies tolerantes ao sombreamento, como aquelas pertencentes às famílias Meliaceae, como G. macrophylla e T. pallida, que tiveram aumentos no número de indivíduos, Myrtaceae (E. blastantha e M. laruotteana) e Lauraceae (O. indecora). A espécie C. urucurana apresentou elevada taxa de mortalidade durante os dois períodos, sendo uma espécie de ciclo de vida curto, com maior ocorrência em locais com maior disponibilidade de luz. Essa substituição está relacionada ao avanço do processo sucessional, em que espécies pioneiras colonizadoras de áreas abertas são substituídas por espécies tardias. Appolinário et al. (2005), Higuchi et al. (2008b) e Miguel et al. (2011) também observaram variações nas taxas de dinâmica as sociadas ao grupo sucessional, com au mento da importância relativa de espécies tardias.

\section{CONCLUS ÕES}

- Apesar de a composição florística e a riqueza permanecerem estáveis durante o período de monitoramento, os resultados demonstraram instabilidade na dinâmica florestal, com grande parte das populações com balanço negativo de abundância (mortalidade superior ao recrutamento) e com redução const ante do número de indivíduos, que podem ser interpretados como sendo a consequência da resposta da floresta aos distúrbios provocados pelas cheias do rio e pela antropização. Ressalte-se que o monitoramento por um período maior de tempo e em outras condições ambientais será necessário para uma melhor compreensão da dinâmica das populações de espécies arbóreas.

\section{REFERÊNCIAS}

APPOLINÁRIO, V.; OLIVEIRA FILHO, A. T.; GUILHERME, F. A. G. Tree population and community dynamics in a Brazilian tropical semideciduous forest. Revista Brasileira de Botânica, São Paulo, v. 28, n. 2, p. 347-360, 2005.

BOTREL, R. T.; YAMAMOTO, K.; RODRIGUES, R. R. Avaliação de um método de análise silvigênica em uma Floresta Estacional Semidecidual. Ciência Florestal, Santa Maria, v. 23, n. 2, p. 391-402, 2013.

BRASIL. Normais climatológicas 1961-1990. Brasília: Ministério da Agricultura e Reforma Agrária. 1992. $84 \mathrm{p}$.

BRODIE, J.; POST, E.; LAURA NCE, W. F. Climate change and tropical biodiversity: a new focus. Trends in Ecology and Evolution, A msterdam, v. 27, n. 3, p. 145-150, 2012.

CARVALHO, F. A.; FELFILI, J. M. Variações tempora is na co munidade arbórea de u ma floresta decidual sobre afloramentos calcários no Brasil Central: composição, estrutura e diversidade florística. Acta Botanica Brasilica, Feira de Santana, v. 25, n. 1, p. 203-214, 2011.

CHAGAS, R. K.; OLIVEIRA FILHO, A. T.; VAN DEN BERG, E.; SCOLFORO, J. R. S. Dinâmica de populações arbóreas em um frag mento de Floresta Estacional Semidecidual Montana em Lavras, Minas Gerais. Revista Árvore, Viçosa, v. 25, n. 1, p. 39-57, 2001.

CORREA, B. S.; VAN DEN BERG, E. Estudo da dinâmica da população de Xylopia brasiliensis Sprengel em relação a parâmetros populacionais e da comunidade em uma floresta de galeria em Itutinga/MG, Brasil. Cerne, Lavras, v. 8, n. 1, p. 1-12, 2002.

COSTA, M. P.; NAPPO, M. E.; CAÇADOR, F. R. D.; BARROS, H. H. D. Avaliação do processo de reabilitação de um trecho de floresta ciliar na bacia do rio Itapemirim/ES. Revista Árvore, Viçosa, v. 34, n. 5, p. $835-851,2010$.

GUILHERME, F. A. G.; OLIVEIRA FILHO, A. T.; APPOLINÁRIO, V.; BEARZOTI, E. Effects of flooding regimes and woody bamboos on tree community dynamics in a section of tropical semideciduous forest in South-Eastern Brazil. Plant Ecology, Nova York, v. 174, n. 1, p. 19-36, 2004.

GUIMARÃES, J. C. C.; VAN DEN BERG, E.; CASTRO, G. C.; MACHADO, E. L. M.; OLIVEIRA FILHO, A. T. Dinâmica do componente arbustivo-arbóreo de uma floresta de galeria aluvial no planalto de Poços de Caldas, MG, Brasil. Revista Brasileira de Botânica, São Pau lo, v. 31, n. 4, p. 621-632, 2008.

HIGUCHI, P.; OliVEIRA FILHO, A. T.; BEBBER, D. P.; BROWN, N. D.; SILVA, A. C. Spatio-temporal patterns of tree community dynamics in a tropical forest fragment in South-East Brazil. Plant Ecology, Nova York, v. 199, n. 1, p. 125-135, 2008a. 
HIGUCHI, P.; OlIVEIRA FILHO, A. T.; SIlVA, A. C.; MACHADO, E. L. M.; SANTOS, R. M.; PIFANO, D. S. Dinâmica da comunidade arbórea em um frag mento de Floresta Estacional Semidecidual Montana em Lav ras, Minas Gerais, em diferentes classes de solos. Revista Árvore, Viçosa, v. 32, n. 3, p. 417-426, $2008 \mathrm{~b}$.

INSTITUTO BRASILEIRO DE GEOGRAFIA E ESTATÍSTICA. Manual técnico da vegetação brasileira. Rio de Janeiro: Instituto Brasileiro de Geografia e Estatística, 2012. 275 p.

KORNING, J.; BALSLEV, H. Growth and mortality of trees in Amazonian tropical rain forest in Ecuador. Journal of Vegetation Science, Hoboken, v. 4, n. 1, p. 77-86, 1994.

LIEBERMAN, D.; LIEBERMAN, M.; PERALTA, R.; HARTSHORN, G. S. Mortality patterns and stand turnover rates in a wet tropical forest in Costa Rica. Journal of Ecology, Hoboken, v. 73, n. 3, p. 915-924, 1985.

LÔBO, D.; LEÃO, T.; MELO, F. P. L.; SANTOS, A. M. M.; TABARELLI, M. Forest fragmentation drives Atlantic forest of northeastern Brazil to biotic homogenization. Diversity and Distributions, Hoboken, v. 17, n. 2, p. 287-296, 2011.

MACHADO, E. L. M.; OLIVEIRA FILHO, A. T. Spatial patterns of tree community dynamics are detectable in a small (4 ha) and disturbed fragment of the Brazilian Atlantic forest. Acta Botanica Brasilica, Feira de Santana, v. 24, n. 1, p. 250-261, 2010.

MASSOCA, P. E. S.; JAKOVAC, A. C. C.; BENTOS, T. V.; WILliAMSON, G. B.; MESQUITA, R. C. G. Dinâmica e trajetórias da sucessão secundária na Amazônia Central. Boletim do Museu Paraense Emílio Goel di. Ciências Naturais, Belém, v. 7, n. 3, p. 235-250, 2012.

MIGUEL, A.; MARIMON, B. S.; OLIVEIRA, E. A.; MARACAHIPES, L.; MARIMON JÚNIOR, B. H. Dinâmica da comunidade lenhosa de uma floresta de galeria na transição Cerrado-Floresta Amazônica no leste de Mato Grosso, em um período de sete anos (1999 a 2006). Biota Neotropica, Campinas, v. 11, n. 1, p. 53-61, 2011.

OLIVEIRA FILHO, A. T.; MELLO, J. M.; SCOLFORO, J. R. Effects of past disturbance and edges on tree community structure and dynamics within a fragment of tropical semideciduous forest in south-eastern Brazil over a five years period (1987-1992). Plant Ecology, Nova York, v. 131, n. 1, p. 45-66, 1997.

OLIVEIRA FILHO, A. T.; CARVALHO, W. A. C.; MACHADO, E. L. M.; HIGUCHI, P.; APPOLINÁRIO, V.; CASTRO, G. C.; SIlvA, A. C.; SANTOS, R. M.; BORGES, L. F.; CORREA, B. S.; BUENO, J. M. A. Dinâmica da comunidade e populações arbóreas da borda e interior de um remanescente florestal na Serra da Mantiqueira, Minas Gerais, em um intervalo de cinco anos (1999-2004). Revista Brasileira de Botânica, São Paulo, v. 30, n. 1, p. 149-161, 2007.

PINTO, J. R. R.; HA Y, J. D. V. Mudanças florísticas e estruturais na comunidade arbórea de uma floresta de vale no Parque Nacional da Chapada dos Guimarães, Mato Grosso, Brasil. Revista Brasileira de Botânica, São Paulo, v. 28, n. 3, p. 523-539, 2005.

PRIMACK, R. B.; ASHTON, P. S.; CHAI LEE, H. S. Growth rates and population structure of Moraceae trees in Sarawak, East Malaysia. Ecology, Ithaca, v. 66, n. 2, p. 577-588, 1985.

R DEVELOPMENT CORE TEAM. R: A language and environment for statistical computing. R Foundation for Statistical Computing, Vienna. Disponível em: <http://www.R-project.org>. Acesso em: 18 jul. 2013.

RIZZINI, C. T. Tratado de fitogeografia do Brasil: aspectos ecológicos, sociológicos e florísticos. Rio de Janeiro: Â mbito Cultural Edições Ltda., 1997. 747 p.

SAITER, F. Z.; GUILHERME, F. A. G.; THOMAZ, L. D.; WENDT, T. Tree changes in a mature rainforest with high diversity and endemis m on the Brazilian cost. Biodi versity and Conservation, Nova York, v. 20, n. 9 , p. 1921-1949, 2011.

SHEIL, D.; MA Y, R. M. Mortality and recruitment rate evaluations in heterogeneous tropical forests. Journal of Ecol ogy, Hoboken, v. 84, n. 1, p. 91-100, 1996.

SILVA, M. R.; ARAÚJO, G. M. Dinâmica da comunidade arbórea de uma floresta semidecidual em Uberlândia, MG, Brasil. Acta Botanica Brasilica, Feira de Santana, v. 23, n. 1, p. 59-56, 2009.

SILVA, A. C.; VAN DEN BERG, E.; HIGUCHI, P.; OLIVEIRA FILHO, A. T.; MARQUES, J. J. G. S. M.; APPOLINÁRIO, V.; PIFANO, D. S.; OGUSUKU, L. M.; NUNES, M. H. Florística e estrutura da comunidade

FLOREST A, Curitiba, PR, v. 46, n. 1, p. 57 - 66, jan. / mar. 2016.

Nunes, M. H. etal.

ISSN eletrônico 1982-4688

DOI: $10.5380 /$ rf.v46il .34782 
arbórea em frag mentos de Floresta Aluvial em São Sebastião da Bela Vista, Minas Ge rais. Revista Brasileira de Botânica, São Paulo, v. 32, n. 2, p. 283-297, 2009.

SILVA, A. C.; HIGUCHI, P.; VAN DEN BERG, E. Effects of soil water table regime on tree community species richness and structure of alluvial forest frag ments in South-East Brazil. Brazilian Journal of Biology, São Carlos, v. 70, n. 3, p. 465-471, 2010.

SILVA, A. C.; VAN DEN BERG, E.; HIGUCHI, P.; NUNES, M. H. Dinâmica da comunidade arbórea após enchente em frag mentos de Floresta Aluvial no sul de Minas Gerais. Revista Árvore, Viçosa, v. 35, n. 4, p. 883893, 2011a.

SILVA, A. C.; HIGUCHI, P.; VAN DEN BERG, E.; NUNES, M. H.; SANTOS, M. C. N. Variação espacial e temporal da dinâmica da comunidade arbórea em frag mentos de Floresta Aluvial no sul de Minas Gerais. Cerne, Lavras, v. 17, n. 4, p. 465-471, 2011 b. 